\title{
Modeling Viral and Drug Kinetics: Hepatitis C Virus Treatment with Pegylated Interferon Alfa-2b
}

\author{
Kimberly A. Powers, B.A., ${ }^{1}$ Narendra M. Dixit, Ph.D., ${ }^{1}$ Ruy M. Ribeiro, Ph.D., ${ }^{1,2}$ \\ Preeti Golia, M.D., ${ }^{3}$ Andrew H. Talal, M.D., ${ }^{3}$ and Alan S. Perelson, Ph.D.1
}

Administration of peginterferon alfa-2b plus ribavirin results in an early hepatitis $\mathrm{C}$ virus (HCV) RNA decay followed by an increase as the drug concentration declines between doses. Upon administration of the next dose 1 week later, the same pattern is observed. We have incorporated pharmacokinetic/pharmacodynamic analysis into a model of viral dynamics to describe the effect that changes in drug concentration and effectiveness can have on viral levels. To illustrate the relationship between pharmacokinetics and viral dynamics, we fit the model to data from four $\mathrm{HCV} /$ human immunodeficiency virus co-infected patients, and obtained good agreement with the measured serum HCV RNA levels. We were able to account for the observed increases in HCV RNA, and estimate virion and drug half-lives that are in agreement with previous reports. Models incorporating pharmacokinetics are needed to correctly interpret viral load changes and estimate drug effectiveness in treatment protocols using peginterferon alfa-2b.

KEYWORDS: PEG-IFN, pharmacokinetics, modeling, HCV, viral dynamics

The delayed clearance of peginterferon alfa- $2 \mathrm{~b}$ (PEG-IFN alfa-2b) from the body allows it to be dosed once weekly in $\mathrm{HCV}$ patients, less frequently than the usual dosing of three times a week for standard IFN alfa. ${ }^{1,2}$ While once-weekly dosing results in greater convenience for patients, it leads to decreases in drug concentration between doses, resulting in decreases in efficacy and increases in viral load as drug concentrations drop. ${ }^{3}$ The effects of the decrease in drug concentration on HCV RNA have not been explored. Explanation of the interplay among these phenomena requires a combination of pharmacokinetic, pharmacodynamic, and viral dynamic models. In this article, we illustrate these principles and present a new theoretical framework to de- scribe the antiviral effectiveness of treatment with PEGIFN alfa-2b plus ribavirin.

\section{VIRAL LOAD DURING TREATMENT WITH PEGYLATED INTERFERON ALFA-2B}

We conducted a preliminary analysis of $4 \mathrm{HCV}$-infected patients treated with $1.5 \mu \mathrm{g} / \mathrm{kg}$ PEG-IFN alfa-2b (12 $\mathrm{kDa})$ once weekly plus daily ribavirin $(13 \mathrm{mg} / \mathrm{kg})$ for 28 days. Three of the 4 patients were male, 3 were infected with genotype $1 \mathrm{a}$ and one with genotype 2, 2 patients were White, one was Hispanic, and one was African American (Table 1). The average patient age was 51 years, and average baseline weight was $86 \mathrm{~kg}$ (Table 1). These

Peginterferons in Hepatitis C Virus: Virological, Pharmacokinetic, and Clinical Implications; Editor in Chief, Paul D. Berk, M.D.; Guest Editor, Emmet B. Keeffe, M.D. Seminars in Liver Disease, volume 23, supplement 1, 2003. Correspondence and reprint requests: Alan S. Perelson, Ph.D., Theoretical Biology and Biophysics, MS K710, Los Alamos National Laboratory, Los Alamos, NM 87545. E-mail: asp@lanl.gov. ${ }^{1 T h e o r e t i c a l ~}$ Biology and Biophysics, Los Alamos National Laboratory, Los Alamos, New Mexico; ${ }^{2}$ Current address: Department of Zoology, University of Oxford, Oxford, United Kingdom; ${ }^{3}$ Department of Medicine and Center for the Study of Hepatitis C, Weill Medical College of Cornell University, New York, New York. Published by Thieme Medical Publishers, Inc., 333 Seventh Avenue, New York, NY 10001, USA. Tel: +1(212) 584-4662. 0272-8087,p;2003,23,s1,013,018,ftx,en;sld00218x. 
Table 1 Patient Demographics

\begin{tabular}{llllll}
\hline Patient & $\begin{array}{l}\text { Age } \\
\text { (years) }\end{array}$ & Sex & Race & $\begin{array}{l}\text { Weight } \\
(\mathbf{k g})\end{array}$ & $\begin{array}{l}\text { HCV } \\
\text { Genotype }\end{array}$ \\
\hline 1 & 56 & $\mathrm{M}$ & Caucasian & 81.7 & $1 \mathrm{a}$ \\
2 & 46 & $\mathrm{M}$ & Hispanic & 88.9 & $1 \mathrm{a}$ \\
3 & 48 & $\mathrm{~F}$ & African-American & 81.6 & $1 \mathrm{a}$ \\
4 & 52 & $\mathrm{M}$ & Caucasian & 93.4 & 2 \\
Average $( \pm \mathrm{SD})$ & $50.5 \pm 4.4$ & & & $86.4 \pm 5.8$ & \\
\hline
\end{tabular}

patients were also co-infected with human immunodeficiency virus (HIV)-1 and had HIV-1 RNA levels below the limit of detection of 400 copies/mL. Serum HCV RNA was measured when the first, second, third, and fifth doses were given, and at regular intervals between doses. We analyzed the viral dynamics during the first week, when sampling was most frequent: at baseline, at 6 and 12 hours after baseline, and on days 1, 2, 3, 5, 6, and 7 after baseline. In patient \#3, no measurements were taken on days 2 and 3, but a measurement was taken on day 4 .

The intensive sampling schedule around the weekly doses of PEG-IFN alfa-2b allowed the characterization of $\mathrm{HCV}$ dynamics as the serum concentration of PEG-IFN alfa-2b peaked and waned. ${ }^{3}$ In most patients, there was an initial fast pronounced decline in HCV RNA following the first dose, the same as had been observed in previous studies of $\mathrm{HCV}$ treatment. ${ }^{4-8}$ A few days after the first dose, most of the patients exhibited a rebound in HCV RNA (Fig. 1). We hypothesize that this rebound resulted from a decline in drug levels and, consequently, in drug effectiveness.

\section{MODELING OF VIRAL DYNAMICS}

The Neumann et $\mathrm{al}^{4}$ model of viral dynamics is described by the following set of differential equations for infected cells and free virus:
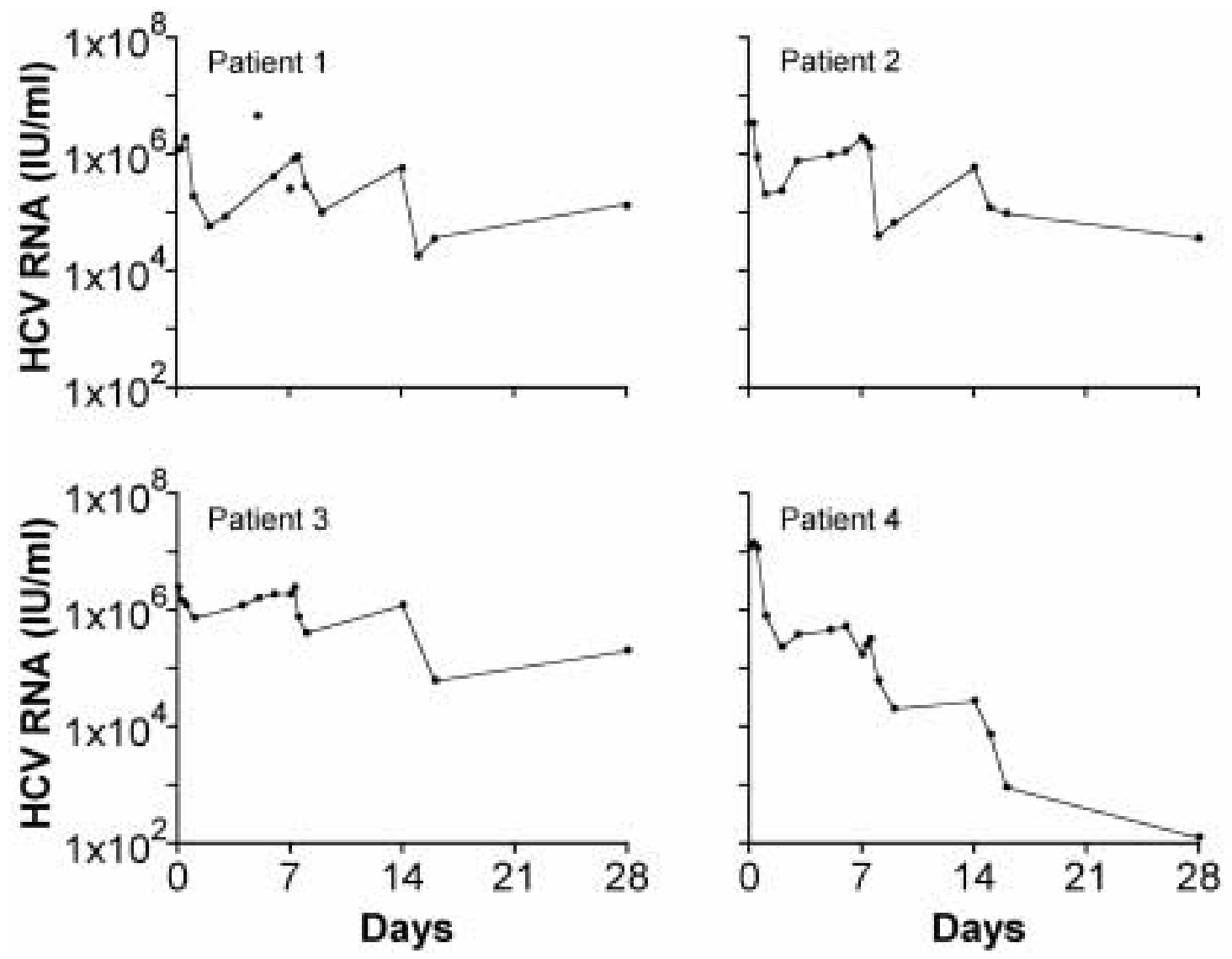

Figure 1 Evolution of serum HCV RNA levels during successive administration of PEG-IFN alfa-2b injections in 4 individuals infected with HCV and HIV. 


$$
\begin{gathered}
d I / d t=\beta V T-\delta I \\
d V / d t=(1-\varepsilon(t)) p I-c V
\end{gathered}
$$

In this system, the free virus, $V$, infects uninfected hepatocytes (the $\beta V T$ term), thereby generating infected cells, $I$, that subsequently produce virus at rate $p$ per cell. The virus is cleared with rate constant $c$ and infected cells are lost at rate $\delta$ per cell. PEG-IFN alfa-2b is assumed to block a fraction, $\varepsilon(t)$, of the production of virus from infected cells but not to affect virion or infected cell clearance rates. To solve these equations, we assume that the number of uninfected cells, $T$, remains approximately constant during therapy.

When drug effectiveness is constant, that is, $\varepsilon(t)=\varepsilon$, these equations can be solved to yield the solution, Equation 2, which predicts that viral load $V(t)$ will decrease with (time on therapy, $t$.

$$
\begin{aligned}
V(t)= & \frac{1}{2} V_{0}\left[\left(1-\frac{c+\delta-2 \varepsilon c}{\theta}\right) e^{-\lambda_{1}\left(t-t_{0}\right)}+\right. \\
& \left.\left(1+\frac{c+\delta-2 \varepsilon c}{\theta}\right) e^{-\lambda_{2}\left(t-t_{0}\right)}\right],
\end{aligned}
$$

where

$$
\begin{gathered}
\lambda_{1}=0.5(c+\delta+\theta), \lambda_{2}=0.5(c+\delta-\theta), \text { and } \\
\theta=\sqrt{(c-\delta)^{2}+4(1-\varepsilon) c \delta} .
\end{gathered}
$$

If $c>>$, then $\lambda_{1} \sim \mathrm{c}$ and $\lambda_{2} \sim \varepsilon \delta$, corresponding to the first and second phase slopes observed when $\ln V(t)$ is plotted against $t$. Additionally, the virus is initially assumed to be in steady state, so that $V_{O}$ represents a constant baseline amount of HCV RNA. The parameter $t_{0}$ describes the delay usually observed between the administration of IFN and its effect in blocking virion production, which causes the initial decline in viral load. When a delay is present, Equation 2 is valid following the delay (i.e., for $t>t_{0}$ ), and for $t<t_{0}, V(t)=V_{0}$. While $V_{o}$ and $t_{0}$ can be roughly estimated directly from the data, here we used nonlinear least-squares regression analysis to fit the first 7 days of viral load data to Equation 2 to estimate $V_{o}$ and $t_{0}$. These values (Table 2) were used in the analyses that follow.

\section{Modeling of Pharmacokinetics- Elimination-Only Model}

While Equation 2 has been shown to accurately fit viral load data obtained when patients are treated daily with high-dose standard IFN, ${ }^{4-6}$ it is not appropriate to describe the changes in viral load when PEG-IFN alfa-2b is given once weekly. With once-weekly dosing there appears to be a reduction in the drug's effectiveness in blocking viral production due to a decline in its concentration between doses, ${ }^{3}$ and hence the assumption of constant drug effectiveness no longer applies. If we assume that after the delay $t_{0}$, the concentration of PEGIFN alfa-2b simply decays exponentially, the drug concentration, $C$, over time can be described by

$$
C(t)=C_{0} e^{-k_{e}\left(t-t_{0}\right)},
$$

where $C_{0}$ is the drug concentration at time $t_{0}$ and $k_{e}$ is the rate constant for drug elimination. Because effectiveness can be assumed to depend on drug concentration as ${ }^{9}$

$$
\varepsilon(t)=\frac{C(t)}{I C_{50}+C(t)},
$$

where $I C_{50}$ is the concentration at which the drug's effectiveness is half its maximum, the decay in drug concentration described in Equation 3 leads to a decrease in effectiveness. Simulated changes in drug concentration and effectiveness, as predicted by this model, are displayed in Figure 2, where we assume that Equation 4 is valid for $t>t_{0}$.

Equations 3 and 4 were incorporated into Equation 1 to create a new model for viral load change under PEG-IFN alfa- $2 \mathrm{~b}$ treatment. The delay $t_{0}$ is interpreted as comprising both the time for the drug level to rise following injection as well as the biological delay be-

\begin{tabular}{|c|c|c|c|c|c|c|c|}
\hline Patient & $\begin{array}{l}V_{0} \\
\left(10^{6} \mathrm{IU} / \mathrm{mL}\right)\end{array}$ & $\begin{array}{l}\delta \\
\left(\text { day }^{-1}\right)\end{array}$ & $\begin{array}{l}c \\
\left(\text { day }^{-1}\right)\end{array}$ & $\epsilon_{\max }$ & $\epsilon_{\min }$ & $\begin{array}{l}k_{e} \\
\left(\text { day }^{-1}\right)\end{array}$ & $\begin{array}{l}t_{0} \\
\text { (days) }\end{array}$ \\
\hline 1 & 1.39 & 0.37 & 4.20 & 0.99 & 0.03 & 1.11 & 0.50 \\
\hline 2 & 3.34 & 0.25 & 5.39 & 0.98 & 0.02 & 1.10 & 0.25 \\
\hline 3 & 2.34 & 0.02 & 1.76 & 0.89 & 0.07 & 0.66 & 0.00 \\
\hline 4 & 12.8 & 0.45 & 9.16 & 0.98 & 0.63 & 0.45 & 0.64 \\
\hline Average $( \pm S D)$ & $5.0 \pm 5.3$ & $0.3 \pm 0.2$ & $5.1 \pm 3.1$ & $0.96 \pm 0.05$ & $0.2 \pm 0.3$ & $0.8 \pm 0.3$ & $0.4 \pm 0.3$ \\
\hline
\end{tabular}
tween IFN binding to its cellular receptor and the antiviral response of the infected cell. Using nonlinear leastsquares regression analysis, data from the first 7 days of treatment were fitted to this "elimination-only model" (Fig. 3). Values for the parameters $c, \delta, C_{0} / I C_{50}$, and $k_{e}$ were estimated, and the maximum effectiveness, that is,

Table 2 Parameter Estimates from Fitting the Elimination-Only Model

The virion production rate, $p$, was fixed at 10 virions/cell/day for all patients. $V_{0}$ and $t_{0}$ were estimated from fits of $\mathrm{HCV}$ RNA to Equation 2. 

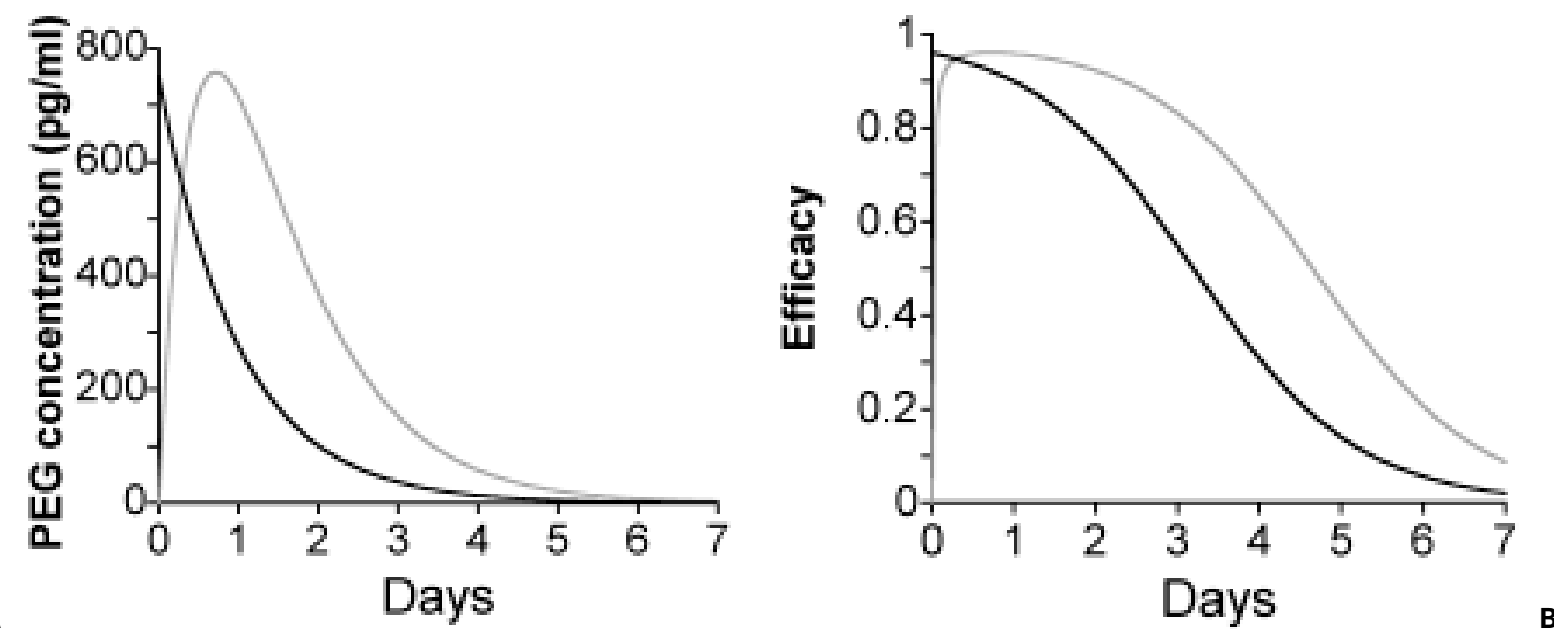

Figure 2 Simulated concentration and efficacy profiles with once-weekly dosing. Delays are not illustrated. Parameter values used in the simulations were: $k_{a}=1.9 \mathrm{~d}^{-1}, k_{e}=1.0 \mathrm{~d}^{-1}$, Dose $=129 \mu \mathrm{g}, 1 C_{50}=30.96 \mathrm{pg} / \mathrm{mL}, C_{0}=750 \mathrm{pg} / \mathrm{mL}$, and $F / V_{d}=0.012 \mathrm{~L}-1$. (A) Drug concentration over time; elimination-only model (black) and absorption and elimination model (grey). (B) Drug efficacy over time; elimination-only model (black) and absorption and elimination model (grey).
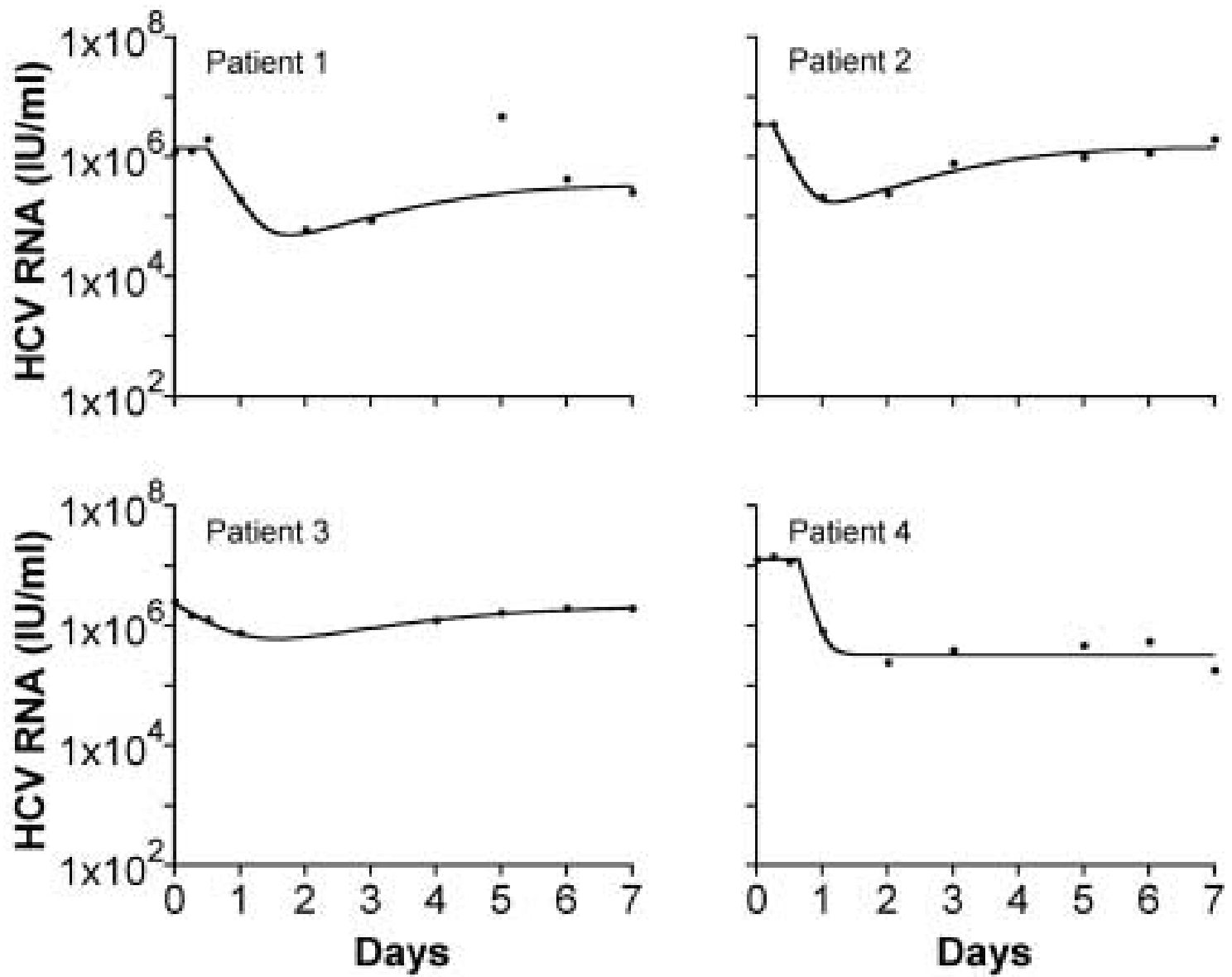

Figure 3 HCV RNA according to the elimination-only model. Best-fit solutions (solid line) of the model, Equations (1) - (4), to experimental data $(\bullet)$ from 4 patients during the first 7 days of treatment. 
$\varepsilon_{\max }=\left(C_{0} / I C_{50}\right) /\left[1+\left(C_{0} / I C_{50}\right)\right]$, was calculated (Table 2). The corresponding average virion and drug half-lives $( \pm$ SD) were $4.6 \pm 3.4$ hours and $23.0 \pm 10.4$ hours, respectively, consistent with previously published values. ${ }^{1,4}$ Virion production was blocked by PEG-IFN alfa-2b with an average maximum effectiveness $\left(\varepsilon_{\max }\right)$ of $96 \%$. In Table 2, we also show the estimated drug efficacy 7 days after the first dose, that is, $\varepsilon_{\text {min }}=\left(C_{0} / \mathrm{IC}_{50}\right)\left(e^{-7 k e)} /[1\right.$ $+\left(C_{0} / I C_{50}\right)\left(e^{\left.-7 k_{\mathrm{e}}\right)}\right)$, just before the second weekly PEGIFN dose. In 3 of the 4 patients studied, this residual effectiveness is very low (<10\%). In P4, the effectiveness even one week post-injection is $63 \%$ and, as Figure 3 illustrates, $\mathrm{P} 4$ was the only patient who did not show an increase in viral load during the week. It is noteworthy that this patient was infected with genotype- $2 \mathrm{HCV}$, for which a higher effectiveness of normal IFN alfa has been reported. ${ }^{5}$

\section{Modeling of Pharmacokinetics- Absorption and Elimination Model}

The "elimination-only model" used above, while simple, does not adequately describe the absorption of drug from the site of subcutaneous injection into the blood and liver. Thus we also conducted analyses with a model that includes both absorption and elimination of PEG-IFN alfa- $2 \mathrm{~b}$ in the blood. ${ }^{9}$

This "absorption and elimination model" describes the change in the amount of drug in the blood $(A)$ as follows:

$$
\frac{d A}{d t}=k_{a} X-k_{e} A,
$$

where $X$ is the amount of drug at the absorption site, $k_{\mathrm{a}}$ is the rate of absorption, and $k_{\mathrm{e}}$ is the rate of elimination. $X$ can be further characterized as $X=F D e^{-k_{a} t}$, where $F$ is the bioavailability (the extent to which the active drug enters systemic circulation) and $D$ is the drug dose. The solution to Equation 5 gives an expression for the amount of drug in the blood over time, $A(t)$, and by dividing this expression by the volume of distribution, $V_{d}$, we obtain the following equation for the concentration of drug in the blood:

$$
C(t)=\frac{k_{a} F D}{\left(k_{e}-k_{a}\right) V_{d}}\left(e^{-k_{a} t}-e^{-k_{e} t}\right),
$$

following a single injection at time $t=0$. More complex expressions are needed when the drug is given multiple times. Again, changes in drug concentration affect effectiveness; however, to incorporate the effects of a delay between PEG-IFN alfa-2b binding its receptor and beginning its biological action, we assume that the effectiveness at time $t$ depends on the drug concentration at time $t-\tau$, that is, there is a delay of length $\tau$ between a cell sensing an IFN concentration $C(t)$ and responding to it. Thus, for $t>\tau$,

$$
\varepsilon(t)=\frac{C(t-\tau)}{I C_{50}+C(t-\tau)} .
$$

Simulated changes in concentration and effectiveness with this model, as described by Equations 6 and 7, are displayed in Figure 2. The absorption and elimination model (Equations 1, 6, and 7) was also fitted to data over the first 7 days of therapy and gave fits comparable to those shown in Figure 3 (data not shown). Conceptually, this model has an advantage over the elimination-only model in that it fully separates pharmacokinetic effects from the delays intrinsic to the interferon response. However, it is more complicated mathematically, has an additional parameter, and requires software specially designed to analyze delay-differential equations.

\section{SUMMARY}

Administration of PEG-IFN alfa-2b plus ribavirin results in early HCV RNA decays in HIV-HCV co-infected patients, followed by viral load increases as the drug concentration and efficacy decline between doses. When the next dose is administered 1 week later, the same pattern is observed. We have used two pharmacokinetic models-one that includes drug elimination only and one that considers both absorption and elimination - to describe changes in concentration and drug effectiveness in blocking virion production during early therapy with PEG-IFN alfa-2b $(12 \mathrm{kDa})$. We have incorporated each of these pharmacokinetic models into the Neumann et $\mathrm{al}^{4}$ model of viral dynamics to describe the effect that changes in drug concentration and effectiveness can have on HCV RNA. In this initial modeling study we did not consider the pharmacokinetics of ribavirin because the drug is administered twice daily. Analyzing data from 4 patients, we obtained good agreement between the models and the measured viral load data, were able to account for the observed increases in HCV RNA, and generated estimates of drug effectiveness in blocking virion production. In contrast to our previous work, ${ }^{4}$ we found that effectiveness is not a constant but changes with time, having a maximum and then decaying until the subsequent dose. Our results suggest that without the modifications suggested here, the Neumann et $\mathrm{al}^{4}$ model-with its assumption of fixed drug effectiveness-is not appropriate for the analysis of treatment data obtained with PEG-IFN $a l f a-2 b$. As these preliminary findings are based on data from only 4 patients, subsequent analyses in larger patient cohorts are warranted to further our understanding of the pharmacokinetics and viral dynamics in patients receiving PEG-IFN alfa- $2 \mathrm{~b}$. 


\section{ACKNOWLEDGMENTS}

Portions of this work were performed under the auspices of the U.S. Department of Energy and were supported by a grant from the Speaker's Fund for Public Health Research awarded by the City of New York, by NIH grants RR06555 (ASP), DK02573 (AHT), by a General Clinical Research Center Grant (M01-RR00047), by Schering-Plough Research Institute, and by a Marie Curie Fellowship from the EU (RMR).

\section{ABBREVIATIONS}

$\mathrm{HCV}$ hepatitis $\mathrm{C}$ virus

HIV human immunodeficiency virus

PEG-IFN pegylated interferon alfa-2b

\section{REFERENCES}

1. Glue P, Fang JW, Rouzier-Panis R, et al. Pegylated interferonalpha 2b: pharmacokinetics, pharmacodynamics, safety, and preliminary efficacy data. Hepatitis C Intervention Therapy Group. Clin Pharmacol Ther 2000;68:556-567

2. Lindsay KL, Trepo C, Heintges T, et al. A randomized, double-blind trial comparing pegylated interferon alfa- $2 \mathrm{~b}$ to inter- feron alfa- $2 b$ as initial treatment for chronic hepatitis C. Hepatology 2001;34:395-403

3. Formann E, Jessner W, Bennett L, Ferenci P. Twice-weekly administration of peginterferon alfa-2b PEG-Intron $(12 \mathrm{kD})$ improves viral kinetics in patients with $\mathrm{HCV}$ genotype 1 . Hepatology 2002;36:288A (Abstract 502)

4. Neumann AU, Lam NP, Dahari H, et al. Hepatitis C viral dynamics in vivo and the antiviral efficacy of interferon- $\alpha$ therapy. Science 1998;282:103-107

5. Neumann AU, Lam NP, Dahari H, et al. Differences in viral dynamics between genotypes 1 and 2 of hepatitis $\mathrm{C}$ virus. J Infect Dis 2000;182:28-35

6. Rosen HR, Ribeiro RR, Weinberger L, et al. Early hepatitis C viral kinetics correlate with long-term outcome in patients receiving high dose induction followed by combination interferon and ribavirin therapy. J Hepatol 2002;37:124-130

7. Zeuzem S, Schmidt JM, Lee JH, Ruster B, Roth WK. Effect of interferon alfa on the dynamics of hepatitis $\mathrm{C}$ virus turnover in vivo. Hepatology 1996;23:366-371

8. Zeuzem S, Herrmann E, Lee JH, et al. Viral kinetics in patients with chronic hepatitis $\mathrm{C}$ treated with standard or peginterferon alpha-2a. Gastroenterology 2001;120:1438-1447

9. Gabrielsson J, Weiner D. Pharmacokinetic and Pharmacodynamic Data Analysis: Concepts \& Application. Stockholm: Swedish Pharmaceutical Press; 2000 